\title{
Duality theorems and topological structures of groups
}

\author{
Nobuhiko Tatsuuma
}

\begin{abstract}
We introduce four different notions of weak Tannaka-type duality theorems, and we define three categories of topological groups, called T-type groups, strongly $T$ type groups, and NOS-groups.

We call a one-parameter subgroup a nontrivial homomorphic image of the additive group $\mathbf{R}$ of real numbers into a topological group $G$. When $G$ does not contain any oneparameter subgroup, we call $G$ a NOS-group.

The aim of this paper is to show the following relations. In the table below, the symbol $\Longleftrightarrow$ means that for a given topological group $G$ the duality theorem on the left-hand side holds if and only if $G$ is of type cited on the right-hand side:
\end{abstract}

(1) u-duality $\Longleftrightarrow$ T-type,

(2) i-duality $\Longleftrightarrow$ strongly T-type,

(3) b-duality $\Longleftrightarrow$ locally compact,

(4) c-duality $\Longleftrightarrow$ locally compact NOS.

We give in the last section some examples which show the actual differences among (1)-(4).

\section{Introduction}

Four types of weak Tannaka-type duality theorems for topological groups are stated as follows.

We take the set $\Omega \equiv\left\{D=\left(\mathcal{H}^{D}, T_{g}^{D}\right)\right\}$ of all unitary representations of given topological group $G$, dimensions of which are bounded by $\max \left(\aleph_{0}, \# G\right)$. Then there exist relations between elements of $\Omega$ as

(1) unitary equivalence: $D_{1} \sim_{W} D_{2}$ (W: intertwining operator),

(2) direct sum: $D_{1} \oplus D_{2}$,

(3) tensor product: $D_{1} \otimes D_{2}$,

(4) contragradient: $D \rightarrow \bar{D}$.

We consider an operator field $\mathbf{A} \equiv\left\{A^{D}\right\}_{D \in \Omega}$ on $\Omega$ satisfying the following:

(B-0) for each $D \in \Omega, A^{D}$ is an operator in a certain category on the representation Hilbert space $\mathcal{H}^{D}$ :

Kyoto Journal of Mathematics, Vol. 54, No. 1 (2014), 75-101

DOI $10.1215 / 21562261-2400283$, (C) 2014 by Kyoto University

Received January 20, 2012. Revised December 3, 2012. Accepted December 4, 2012.

2010 Mathematics Subject Classification: Primary 22A25; Secondary 22D35. 
(B-1) $D_{1} \sim_{W} D_{2} \Longrightarrow W A^{D_{1}} W^{-1}=A^{D_{2}}$,

(B-2) $A^{D_{1}} \oplus A^{D_{2}}=A^{D_{1} \oplus D_{2}}$,

(B-3) $A^{D_{1}} \otimes A^{D_{2}}=A^{D_{1} \otimes D_{2}}$,

(B-4) $\overline{A^{D}}=A^{\bar{D}}$.

In the condition (B-0), for the terminology certain category we consider four cases as follows.

At first, we take the unitarity property, that is,

(B-01) for each $D \in \Omega, A^{D}$ is a unitary operator on the representation Hilbert space $\mathcal{H}^{D}$.

We shall call such an operator field which satisfies (B-01)-(B-4) simply a birepresentation of $G$ and write $\mathcal{U}$ for the set of all birepresentations.

Secondly, we take isometricity, that is,

(B-02) for each $D \in \Omega, A^{D}$ is an isometric operator on the representation Hilbert space $\mathcal{H}^{D}$.

We shall call such an operator field which satisfies (B-02), (B-1), (B-2), (B-3), and (B-4) an isobirepresentation of $G$ and write $\mathcal{J}$ for the set of all isobirepresentations.

Thirdly, we take nonzero and uniform boundedness, that is,

(B-03) for each $D \in \Omega, A^{D}$ is a nonzero bounded operator such that $\left\|A^{D}\right\| \leq 1$ on the representation Hilbert space $\mathcal{H}^{D}$.

We shall call such an operator field which satisfies (B-03), (B-1), (B-2), (B-3), (B-4) a bd-birepresentation of $G$ and write $\mathcal{B}$ the set of all bd-birepresentations.

Lastly, we take the property nonzero closed with a common fixed domain $\mathcal{D}^{D}$ dense in $\mathcal{H}^{D}$ and range in the same subspace for each $D$.

(B-04) For each $D \in \Omega, A^{D}$ is a nonzero closed operator on $\mathcal{H}^{D}$ with domain and range a fixed dense subspace $\mathcal{D}^{D}$ in common.

But in this case, we must assume relations for subspaces $\mathcal{D}^{D}$ as

(B-041) $\mathcal{D}^{D_{1}} \sim_{W} \mathcal{D}^{D_{2}} \Longrightarrow W \mathcal{D}^{D_{1}}=\mathcal{D}^{D_{2}}$,

(B-042) $\mathcal{D}^{D_{1}} \oplus \mathcal{D}^{D_{2}} \subset \mathcal{D}^{D_{1} \oplus D_{2}}$

$(\mathrm{B}-043) \mathcal{D}^{D_{1}} \otimes \mathcal{D}^{D_{2}} \subset \mathcal{D}^{D_{1} \otimes D_{2}}$,

(B-044) $\overline{\mathcal{D}}^{D}=\mathcal{D}^{\bar{D}}$.

We shall call such an operator field that satisfies (B-04), (B-1), (B-2), (B-3), (B-4) a cl-birepresentation of $G$ and write $\mathcal{C}$ for the set of all cl-birepresentations.

Of course, a birepresentation is an isobirepresentation, an isobirepresentation is a bd-birepresentation, and a bd-birepresentation is a cl-birepresentation. 
PROPOSITION 0.1

We have

$$
\mathcal{U} \subset \mathcal{J} \subset \mathcal{B} \subset \mathcal{C}
$$

On the space $\mathcal{B}$, we give a topology which is the product of weak topologies $\tau^{D}$ on each spaces $\mathcal{B}^{D}$ of bounded operators on Hilbert spaces $\mathcal{H}^{D}$. We write this topology on $\mathcal{B}$ as $\tau$. The topologies on $\mathcal{U}, \mathcal{J}$ are the restriction of $\tau$ onto each of the spaces.

In the case of cl-birepresentations, we must consider for $\mathcal{C}^{D}$ the space of closed operators with domain $\mathcal{D}^{D}$ at each component $D$, the topology which is the weakest topology $\tau_{\mathcal{C}}^{D}$ making continuous all matrix elements $\left\langle A^{D} v^{D}, u^{D}\right\rangle$ $\left(v^{D}, u^{D} \in \mathcal{D}^{D}\right)$, and give the product topology $\tau_{C}$ on the space $\mathcal{C}$.

\section{PROPOSITION 0.2}

Topology $\tau$ is the restriction of $\tau_{\mathcal{C}}$ to the space $\mathcal{B}$ from $\mathcal{C}$.

\section{Proof}

For any fixed $D$ and $v^{D}, u^{D}$ in $\mathcal{D}^{D},\left\langle A^{D} v^{D}, u^{D}\right\rangle$ is a continuous function of $A^{D}$ with respect to the weak topology $\tau^{D}$ on the space $\mathcal{B}^{D}$.

Therefore it is sufficient to show for any $v^{D}, u^{D}\left(\left\|v^{D}\right\|=\left\|u^{D}\right\|=1\right)$ in $\mathcal{H}^{D}$, that the function $\left\langle A^{D} v^{D}, u^{D}\right\rangle$ is continuous with respect to $\left.\tau_{\mathcal{C}}^{D}\right|_{\mathcal{B}^{D}}$.

The common fixed domain $\mathcal{D}^{D}$ is dense in $\mathcal{H}^{D}$ for each $D$, and we assumed by (B-03) that the components of $\mathcal{B}^{D}$ are bounded by 1 . So for any $\varepsilon>0$ as in (B-03), there exist $v_{0}^{D}, u_{0}^{D}\left(\left\|v_{0}^{D}\right\|=\left\|u_{0}^{D}\right\|=1\right)$ in $\mathcal{D}^{D}$ such that

$$
\left\|v^{D}-v_{0}^{D}\right\|<\varepsilon, \quad\left\|u^{D}-u_{0}^{D}\right\|<\varepsilon .
$$

This shows for any $A^{D}$ bounded as $\left\|A^{D}\right\| \leq 1$,

$$
\begin{aligned}
& \left|\left\langle A^{D} v^{D}, u^{D}\right\rangle-\left\langle A^{D} v_{0}^{D}, u_{0}^{D}\right\rangle\right| \\
& \quad \leq\left|\left\langle A^{D} v^{D}-A^{D} v_{0}^{D}, u^{D}\right\rangle\right|+\left|\left\langle A^{D} v_{0}^{D}, u^{D}-u_{0}^{D}\right\rangle\right| \\
& \quad \leq\left\|A^{D} v^{D}-A^{D} v_{0}^{D}\right\| \times\left\|u^{D}\right\|+\left\|A^{D} v_{0}^{D}\right\| \times\left\|u^{D}-u_{0}^{D}\right\| \\
& \quad \leq\left\|A^{D}\right\| \times\left\|v^{D}-v_{0}^{D}\right\|+\left\|A^{D}\right\| \times\left\|u^{D}-u_{0}^{D}\right\| \leq \varepsilon .
\end{aligned}
$$

Therefore on $\mathcal{B}^{D}$ the function $\left\langle A^{D} v^{D}, u^{D}\right\rangle$ is continuous with respect to $\tau_{\mathcal{C}}^{D}$ as a limit of uniform convergence of continuous functions $\left\langle A^{D} v_{0}^{D}, u_{0}^{D}\right\rangle$.

For any $g \in G$ the operator field $\mathbf{T}_{g} \equiv\left\{T_{g}^{D}\right\}_{D \in \Omega}$ gives a birepresentation.

Our weak Tannaka-type duality theorems assert the converses, which are separated into the set theoretical part and the topological part.

\section{SET PART OF U-DUALITY'S ASSERTION}

For any birepresentation $\mathbf{U} \equiv\left\{U^{D}\right\}_{D \in \Omega}$, there exists a unique $g \in G$ such that $U^{D}=T_{g}^{D} \quad(\forall D \in \Omega)$. 
SET PART OF I-DUALITY'S ASSERTION

For any isobirepresentation $\mathbf{J} \equiv\left\{J^{D}\right\}_{D \in \Omega}$, there exists a unique $g \in G$ such that $J^{D}=T_{g}^{D}(\forall D \in \Omega)$.

\section{SET PART OF B-DUALITY'S ASSERTION}

For any bd-birepresentation $\mathbf{B} \equiv\left\{B^{D}\right\}_{D \in \Omega}$, there exists a unique $g \in G$ such that $B^{D}=T_{g}^{D}(\forall D \in \Omega)$.

\section{SET PART OF C-DUALITY'S ASSERTION}

For any cl-birepresentation $\mathbf{C} \equiv\left\{C^{D}\right\}_{D \in \Omega}$, there exists a unique $g \in G$ such that $C^{D}=T_{g}^{D}(\forall D \in \Omega)$.

\section{TOPOLOGICAL ASSERTION OF THESE DUALITIES}

Moreover, the topologies given above coincide with the original topology of $G$ under the correspondence $g \rightarrow \mathbf{T}_{g}$.

PROPOSITION 0.3

$$
\text { c-duality } \Longrightarrow \text { b-duality } \Longrightarrow \text { i-duality } \quad \Longrightarrow \quad u \text {-duality. }
$$

\section{Proof}

From Proposition 0.1, the set part of this implication is valid. Proposition 0.2 shows that the topological part is also satisfied.

\section{NOTATION}

For a representation $D \equiv\left\{\mathcal{H}^{D}, T_{g}^{D}\right\}$ of $G$, we take its cyclic subrepresentation on the closed subspace $\left(\mathcal{H}^{D}\right)$ of $\mathcal{H}^{D}$ spanned by $\left\{T_{g}^{D} v^{D}\right\}_{g \in G}$ for a fixed $v^{D} \in$ $\mathcal{H}^{D},\left\|v^{D}\right\|=1$. We express it as $(D)=\left\{\left(\mathcal{H}^{D}\right), T_{g}^{D}, v^{D}\right\}$.

Hereafter, for two cyclic representations $D_{j}=\left\{\mathcal{H}^{j}, T_{g}^{j}, v^{j}\right\}, j=1,2$, we denote cyclic subrepresentations contained in $D_{1} \oplus D_{2}$ and $D_{1} \otimes D_{2}$, respectively, as

$$
\begin{aligned}
& \left(D_{1} \oplus D_{2}\right) \equiv\left\{\left(\mathcal{H}^{1} \oplus \mathcal{H}^{2}\right), T_{g}^{1} \oplus T_{g}^{2}, v^{1} \oplus v^{2}\right\}, \\
& \left(D_{1} \otimes D_{2}\right) \equiv\left\{\left(\mathcal{H}^{1} \otimes \mathcal{H}^{2}\right), T_{g}^{1} \otimes T_{g}^{2}, v^{1} \otimes v^{2}\right\} .
\end{aligned}
$$

In Section 1 of this paper, we give the notion of an SSUR of $G$, and "complete" and "b-complete" properties for $G$, after [8, Section 2]. Using these concepts, we define categories of T-type and strongly T-type groups for topological groups.

Section 2 is devoted to proving the condition $\left(\mathrm{W}-3^{\prime}\right)$ in $[8$, Section 8], for topological groups $G$ to have an SSUR. This shows that a T-type group is a well-behaved group, and a strongly T-type group is strongly well behaved as is stated in [8, Sections 7, 8]. 
We show in Section 3 that a T-type group satisfies weak, Tannaka-type i-duality.

Since strongly T-type deduces T-type, this gives also the following.

(1) A T-type group satisfies u-duality.

(2) A strongly-T-type group satisfies i-duality.

The converse problems for T-type groups and strongly T-type groups are solved in Section 4. And we obtain the following:

(3) if a topological group $G$ satisfies u-duality, then $G$ is T-type;

(4) if a topological group $G$ satisfies i-duality, then $G$ is strongly T-type.

We discuss in Section 5 the similar problems for locally compact groups and locally compact NOS groups.

Summarizing the above results, we state the main theorem in Section 6 .

We give in the same section several examples of groups of each type.

\section{Separating systems of unitary representations: T-type groups and strongly T-type groups}

We consider a Hausdorff (i.e., $T_{2^{-}}$) topological group $G$.

\section{DEFINITION 1.1}

We say that a set $\Omega_{0} \equiv\left\{D_{\alpha} \equiv\left\{\mathcal{H}^{D_{\alpha}}, T_{g}^{D_{\alpha}}, v^{D_{\alpha}}\right\}\right\}_{\alpha \in A}$ of cyclic unitary representations of $G$ gives a separating system of unitary representations (SSUR) if for any neighborhood $V$ of the unit $e$ in $G$, there exist a positive definite function $\eta^{D}(g) \equiv\left\langle T_{g}^{D} v^{D}, v^{D}\right\rangle\left(D \in \Omega_{0},\left\|v^{D}\right\|=1\right)$ and $\varepsilon>0$ such that

$$
F(D, \varepsilon) \equiv\left\{g \in G|| 1-\eta^{D}(g) \mid<\varepsilon\right\} \subset V .
$$

For any given cyclic unitary representation $D \equiv\left\{\mathcal{H}^{D}, T_{g}^{D}, v^{D}\right\}\left(\left\|v^{D}\right\|=1\right)$, and the trivial representation $I=\left\{\mathbf{C}, I_{g}, v_{0}\right\}$ of $G$, we define a unitary representation $D_{p} \equiv I \oplus D \oplus \bar{D}$ and its cyclic part $\left(D_{p}\right)$, whose representation space is spanned by the vector

$$
v_{p} \equiv\left(2^{-1 / 2}\right) v_{0} \oplus(1 / 2)\left(v^{D} \oplus v^{\bar{D}}\right) .
$$

Here $\bar{D} \equiv\left\{\mathcal{H}^{\bar{D}}, T_{g}^{\bar{D}}, v^{\bar{D}}\right\}$ is the contragradient representation of $D$ and $v^{\bar{D}}$ is the vector in the space $\mathcal{H}^{\bar{D}}$ corresponding to $v^{D}$. We showed in our previous paper (see [8, Corollaries 1-2-1, 1-2-2]) that

$$
1 \geq\left\langle T_{g}^{D_{p}} v_{p}, v_{p}\right\rangle \geq 0 .
$$

For any $D$ in $\Omega$ we write $\eta^{D}(g) \equiv\left\langle T_{g}^{D} v^{D}, v^{D}\right\rangle$ and

$$
F(D, \varepsilon) \equiv\left\{g \in G|| 1-\eta^{D}(g) \mid<\varepsilon\right\} ;
$$

then

$$
1>\forall \varepsilon \geq 0, \exists \delta>0, \quad F\left(\left(D_{p}\right), \delta\right) \subset F(D, \varepsilon) .
$$


Using (1.3), if a topological group $G$ has an SSUR $\Omega_{0} \equiv\left\{D_{\alpha}\right\}_{\alpha \in A}$, then we can select a new SSUR $\Omega_{1} \equiv\left\{\left(D_{\alpha, p}\right)_{\alpha \in A}\right\}$, for which any $\left(D_{\alpha, p}\right)=\left\{\left(\mathcal{H}^{D_{\alpha, p}}\right)\right.$, $\left.T_{g}^{D_{\alpha, p}}, v^{D_{\alpha, p}}\right\} \in \Omega_{1}$ has a nonnegative-valued positive definite function $\eta^{D_{\alpha, p}}(g) \equiv$ $\left\langle T_{g}^{D_{\alpha, p}} v^{D_{\alpha, p}}, v^{D_{\alpha, p}}\right\rangle$.

For a given Hilbert space $\mathcal{H}$, we denote by $B(\mathcal{H})$ the space of all bounded operators on $\mathcal{H}$, by $U(\mathcal{H})$ the space of all unitary operators on $\mathcal{H}$, and by $J(\mathcal{H})$ the space of all isometric operators on $\mathcal{H}$. Put the weak topologies on each of the spaces.

For any isometric operator $J$ on a Hilbert space $\mathcal{H}$, we get

$$
\begin{aligned}
\|J v-v\|^{2} & =\|J v\|^{2}+\|v\|^{2}-2 \Re\langle J v, v\rangle \\
& =2(\langle v, v\rangle-\Re(\langle J v, v\rangle))=2 \Re(\langle v-J v, v\rangle) .
\end{aligned}
$$

This shows that on $J(\mathcal{H})$, and so on $U(\mathcal{H})$ too, the weak topology coincides with the strong topology.

Moreover, $U(\mathcal{H})$ becomes a topological group with the multiplication of operators and this topology. As a group topology, this topology gives a uniform structure on $U(\mathcal{H})$.

For a topological group $G$, let $D \equiv\left\{\mathcal{H}^{D}, T_{g}^{D}\right\}$ be any unitary representation. Then the map $G \ni g \rightarrow T_{g}^{D} \in U\left(\mathcal{H}^{D}\right) \subset J\left(\mathcal{H}^{D}\right)$ is continuous for each $D$.

Construct $\mathbf{U}(\Omega) \equiv \prod_{D \in \Omega} U\left(\mathcal{H}^{D}\right) \subset \mathbf{J}(\Omega) \equiv \prod_{D \in \Omega} J\left(\mathcal{H}^{D}\right)$ with natural product topologies. The maps

$$
\begin{aligned}
& G \ni g \longmapsto\left(T_{g}^{D}\right)_{D \in \Omega} \in \mathcal{U} \quad\left(\subset \prod_{D \in \Omega} U\left(\mathcal{H}^{D}\right)=\mathbf{U}(\Omega)\right), \\
& G \ni g \longmapsto\left(T_{g}^{D}\right)_{D \in \Omega} \in \mathcal{U} \subset \mathcal{J} \subset \prod_{D \in \Omega} J\left(\mathcal{H}^{D}\right)=\mathbf{J}(\Omega)
\end{aligned}
$$

are into-homomorphisms as topological groups.

When $G$ is a $T_{2}$-topological group with an SSUR [7, Lemma 1.4] shows that the map (1.5) is an into-isomorphism, so by this map, $G$ is embedded as a topological group in $\mathbf{J}(\Omega)$.

We denote by $G_{J}$ the image of $G$ under the map (1.6) into $\mathbf{J}(\Omega)$.

LEMMA 1.1

Let $G$ be a $T_{2}$-topological group with an SSUR.

(1) The weak Tannaka-type u-duality theorem is valid for $G$, if and only if $G_{J}=\mathcal{U}$ and the map (1.5) is an isomorphism between $G$ and its image $G_{J}$ in $\mathbf{U}(\Omega)$ as topological groups.

(2) The weak Tannaka-type i-duality theorem is valid for $G$, if and only if $G_{J}=\mathcal{J}$ and the map (1.6) is an isomorphism between $G$ and its image $G_{J}$ in $\mathbf{J}(\Omega)$ as topological spaces. In this case, $G_{J}=\mathcal{U}=\mathcal{J}$.

Proof

It is obvious from the definitions. 
In [8, Section 2, Definition 2.1], we defined the notions of l-Cauchy and b-Cauchy properties for a filter base as follows.

\section{DEFINITION 1.2}

A filter base $\mathcal{F} \equiv\left\{F_{\alpha}\right\}_{\alpha \in \Gamma}$ on $G$, where $\Gamma$ is a partially ordered set, is called l-Cauchy (hereafter simply Cauchy), if for any neighborhood $V$ of $e \in G$, there exists an $\alpha \in \Gamma$ such that

$$
\forall \beta, \gamma \succ \alpha(\beta, \gamma \in \Gamma), \quad F_{\beta}^{-1} F_{\gamma} \subset V .
$$

We say that $\mathcal{F}$ is $b$-Cauchy (both Cauchy) when both of $\mathcal{F}$ and $\mathcal{F}^{-1} \equiv\left\{F_{\alpha}^{-1}\right\}_{\alpha \in \Gamma}$ are Cauchy at the same time.

If any Cauchy (resp., $b$-Cauchy) filter base has limit points in $G$, we say that $G$ is complete (resp., $b$-complete).

Evidently a $b$-Cauchy filter base is also Cauchy, so a complete group is $b$-complete too.

If a filter base $\mathcal{F}$ converges to a point $g_{0}$ in $G$, then $\mathcal{F}^{-1}$ converges to $g_{0}^{-1}$.

We consider the topological group $\mathbf{G} \equiv \mathbf{U}(\Omega)=\prod_{D \in \Omega} U\left(\mathcal{H}^{D}\right)$ and a Cauchy (resp., b-Cauchy) filter base $\mathcal{F} \equiv\left\{F_{\alpha}\right\}$ on $\mathbf{G}$. Projection image $\mathcal{F}^{D} \equiv\left\{F_{\alpha}^{D} \equiv\right.$ $\left.\operatorname{Proj}_{\mathcal{H}^{D}} F_{\alpha}\right\}$ for any $D \in \Omega$ gives a Cauchy (resp., $b$-Cauchy) filter base on $U\left(\mathcal{H}^{D}\right)$.

Conversely, for a filter base $\mathcal{F} \equiv\left\{F_{\alpha}\right\}_{\alpha \in \Gamma}$ on $\mathbf{U}(\Omega)$ to be Cauchy (resp., $b$-Cauchy), it is enough that, for any $D$ in $\Omega, \mathcal{F}^{D}$ are Cauchy (resp., $b$-Cauchy).

Since on $\mathbf{U}(\Omega)$ the weak topology is equivalent to the strong topology, we can consider these Cauchy or $b$-Cauchy properties in the sense of strong topology on $\mathbf{U}(\Omega)$.

Let $\mathcal{F} \equiv\left\{F_{\alpha}\right\}_{\alpha \in \Gamma}$ be a Cauchy filter base on $\mathbf{U}(\Omega)$.

For any $v \in \mathcal{H}^{D}$ for a fixed $D$, a Cauchy filter base $\left\{F_{\alpha}^{D} v\right\}_{\alpha \in \Gamma}$ converges to a vector $u(v)$ in the Hilbert space $\mathcal{H}^{D}$, that is, for any $U_{\alpha}^{D} \in F_{\alpha}^{D}$ and any $v \in \mathcal{H}^{D}$, strong- $\lim _{\alpha} U_{\alpha}^{D} v=u(v)$. Then, for any $a, b \in \mathbf{C}$,

$$
\lim _{\alpha} U_{\alpha}^{D}\left(a v_{1}+b v_{2}\right)=a u\left(v_{1}\right)+b u\left(v_{2}\right), \quad\|u(v)\|=\lim _{\alpha}\left\|U_{\alpha}^{D} v\right\|=\|v\| .
$$

Therefore the map $\mathcal{H}^{D} \ni v \rightarrow u(v) \in \mathcal{H}^{D}$ is linear and isometric. Thus there exists an isometric operator $B^{D}$ such that $u(v)=B^{D} v$.

\section{LEMMA 1.2}

Any Cauchy filter base on $\mathbf{U}(\Omega)=\prod_{D \in \Omega} U\left(\mathcal{H}^{D}\right)$ converges to a $\mathbf{B} \equiv\left(B^{D}\right)_{D \in \Omega} \in$ $\mathbf{J}(\Omega)=\prod_{D \in \Omega} J\left(\mathcal{H}^{D}\right)$, where $B^{D}$ are isometric operators.

For a topological group $G$, any filter base $\mathcal{F}$ on it is mapped to a filter base $\mathcal{F}_{J}$ in $G_{J}$. And if $\mathcal{F}$ is Cauchy (resp., b-Cauchy), then $\mathcal{F}_{J}$ in $\mathbf{U}(\Omega)$ is also Cauchy (resp., b-Cauchy).

\section{LEMMA 1.3}

(1) A Cauchy filter base $\mathcal{F}_{J}$ on a group $G_{J}$ converges to an element $\mathbf{B} \equiv$ $\left(B^{D}\right)_{D \in \Omega}$ in $\mathbf{J}(\Omega)$. 
(2) If $\mathcal{F}_{J}$ is b-Cauchy, then $\mathcal{F}_{J}$ converges to an element in $\mathbf{U}(\Omega)$.

Proof

(1) The result is deduced directly from Lemma 1.2.

(2) If $\mathcal{F}_{J}$ is $b$-Cauchy, then $\mathcal{F}_{J}$ and $\mathcal{F}_{J}^{-1}$ are both Cauchy. So by Lemma 1.2, $\mathcal{F}_{J}$ converges to a $\mathbf{B} \equiv\left(B^{D}\right)_{D \in \Omega} \in \mathbf{J}(\Omega)$ and $\mathcal{F}_{J}^{-1}$ converges to a $\mathbf{C} \equiv\left(C^{D}\right)_{D \in \Omega} \in$ $\mathbf{J}(\Omega)$, where $B^{D}$ and $C^{D}$ are isometric operators. This means that for each $D \in \Omega$, the component $\mathcal{F}_{J}^{D} \equiv\left\{F_{\alpha}^{D}\right\}$ of $\mathcal{F}_{J}$ converges to $B^{D}$ and $\left(\mathcal{F}^{-1}\right)_{J}^{D} \equiv\left\{\left(F_{\alpha}^{D}\right)^{-1}\right\}$ of $\mathcal{F}_{J}^{-1}$ converges to $C^{D}$ under the strong topology of $J\left(\mathcal{H}^{D}\right)$, so that, for any neighborhood $V^{D}$ of $I^{D}$ (the identity operator in $\left.J\left(\mathcal{H}^{D}\right)\right), B^{D} \in F_{\alpha}^{D} V^{D}$ and $C^{D} \in V^{D}\left(F_{\alpha}^{D}\right)^{-1}$.

For given neighborhood $W^{D}$ of $I^{D}$ in $J\left(\mathcal{H}^{D}\right)$, take $V^{D}$ as $\left(V^{D}\right)^{3} \subset W^{D}$, and take $\alpha$ as $F_{\alpha}^{-1} F_{\alpha} \subset V^{D}$. Then $C^{D} B^{D} \in V^{D} F_{\alpha}^{-1} F_{\alpha} V^{D} \subset\left(V^{D}\right)^{3} \subset W^{D}$, that is, $C^{D} B^{D} \in \bigcap W^{D}$; here $W^{D}$ runs any neighborhood of $I^{D}$.

We get $C^{D} B^{D}=I^{D}$.

But $C^{D}$ is an isometric operator having the range the full space $\mathcal{H}^{D}$ and therefore is a unitary operator on $\mathcal{H}^{D}$.

Therefore we have

$$
\forall D \in \Omega, \quad B^{D}=\left(C^{D}\right)^{-1} .
$$

Thus $B^{D}$ must be a unitary operator, and $\mathbf{B} \equiv\left(B^{D}\right)_{D \in \Omega} \in \mathbf{U}(\Omega)$.

\section{DEFINITION 1.3}

(1) We say that a topological group $G$ is T-type if

(T-1) $G$ has an SSUR (separating condition), and

(T-2) $G$ is $b$-complete.

(2) We say that a topological group $G$ is strongly T-type if

(T-1) $G$ has an SSUR (separating condition), and

$\left(\mathrm{T}-2^{\prime}\right) G$ is complete.

Since a complete group is $b$-complete, so a strongly T-type group is a T-type group.

\section{REMARK}

The conditions (T-1), (T-2), and (T-2') above are just the same as the conditions $(\mathrm{W}-1),(\mathrm{W}-2)$, and $\left(\mathrm{W}-2^{\prime}\right)$ defined in $[8$, Sections 7,8$]$, respectively.

In the succeeding sections, we shall discuss relations between groups of this type and two types of weak Tannaka-type dualities.

We showed in [8, Lemma 7.1] that any locally compact groups and any closedtype inductive limits of such groups are strongly T-type, therefore of T-type. 


\section{Birepresentation and isobirepresentation of $G$}

Now we discuss some elementary properties of isobirepresentations on $G$ with an SSUR. Since any birepresentation is also an isobirepresentation, these properties are valid for it too.

The following arguments in Lemma 2.1 and its corollaries are just similar to those of [7, Section 6]. The only difference is to change the letter $U$ to $J$; we will repeat it to confirm.

The condition (B-4) assures that for any isobirepresentation $\mathbf{J} \equiv\left\{J^{D}\right\}$,

$$
J^{\bar{D}}=\overline{\left(J^{D}\right)}
$$

\section{LEMMA 2.1}

For $D^{0} \equiv D \oplus \bar{D}$, the matrix element $\left\langle J^{D^{0}}(u \oplus \bar{u}), v \oplus \bar{v}\right\rangle$ is real valued.

Proof

$\left\langle J^{D^{0}}(u \oplus \bar{u}), v \oplus \bar{v}\right\rangle=\left\langle J^{D} u, v\right\rangle+\left\langle J^{\bar{D}} \bar{u}, \bar{v}\right\rangle=\left\langle J^{D} u, v\right\rangle+\left\langle\overline{\left(J^{D} u\right)}, \bar{v}\right\rangle=\left\langle J^{D} u, v\right\rangle+$ $\overline{\left\langle J^{D} u, v\right\rangle} \in \mathbf{R}$.

\section{COROLLARY 2.1.1}

Put $D_{p} \equiv I \oplus D \oplus \bar{D}$. Take vectors $w_{0} \in \mathcal{H}^{I}, w \in \mathcal{H}^{D}$ such that $2^{1 / 2}\left\|w_{0}\right\|=2\|w\|=$ 1 , and put $v_{p} \equiv w_{0} \oplus w \oplus \bar{w}$. Then the matrix element

$$
\left\langle J^{D_{p}} v_{p}, v_{p}\right\rangle=\left\langle J^{D_{p}}\left(w_{0} \oplus w \oplus \bar{w}\right), w_{0} \oplus w \oplus \bar{w}\right\rangle \geq 0 .
$$

Proof

We have

$$
\begin{aligned}
\left\langle J^{D_{p}} v_{p}, v_{p}\right\rangle & =\left\langle\left(I w_{0}\right) \oplus\left(J^{D} w\right) \oplus\left(J^{\bar{D}} \bar{w}\right), w_{0} \oplus w \oplus \bar{w}\right\rangle \\
& =\left\langle w_{0}, w_{0}\right\rangle+\left\langle J^{D} w, w\right\rangle+\left\langle J^{\bar{D}} \bar{w}, \bar{w}\right\rangle \\
& =\left\|w_{0}\right\|^{2}+2 \Re\left\langle J^{D} w, w\right\rangle \\
& =2^{-1}+2 \Re\left\langle J^{D} w, w\right\rangle .
\end{aligned}
$$

But $\left|\left\langle J^{D} w, w\right\rangle\right| \leq\|w\|^{2}=2^{-2}$. So $-2^{-1} \leq 2 \Re\left\langle J^{D} w, w\right\rangle \leq 2^{-1}$, whence $\left\langle J^{D_{p}} w_{p}, w_{p}\right\rangle \geq 0$.

COROLLARY 2.1.2

As in the case of Corollary 2.1.1, for $D_{p} \equiv I \oplus D \oplus \bar{D}$,

$$
\forall g \in G, \quad\left\langle T_{g}^{D_{p}} J^{D_{p}} v_{p}, v_{p}\right\rangle \geq 0 \quad\left(v_{p}=w_{0} \oplus w \oplus \bar{w}\right) .
$$

Proof

For any isobirepresentations $\mathbf{J} \equiv\left\{J^{D}\right\}$ and $\mathbf{T}_{g} \equiv\left\{T_{g}^{D}\right\}, \mathbf{T}_{g} \mathbf{J} \equiv\left\{T_{g}^{D} J^{D}\right\}$ is also an isobirepresentation. So we can apply the result of Corollary 2.1.1. 
Let $D=\left\{\mathcal{H}^{D}, T_{g}^{D}, v^{D}\right\}$ be a cyclic unitary representation of $G$. Denote by $\eta^{D}(g) \equiv$ $\left\langle T_{g}^{D} v^{D}, v^{D}\right\rangle$ the positive definite function to which $D$ belongs, and put

$$
K^{D}(g) \equiv\left\langle T_{g}^{D} J^{D} v^{D}, v^{D}\right\rangle .
$$

\section{LEMMA 2.2}

Let $\mathbf{J}$ be an isobirepresentation. Then, for any $D \equiv\left\{\mathcal{H}^{D}, T_{g}^{D}, v^{D}\right\} \quad\left(\left\|v^{D}\right\|=1\right)$ in $\Omega$,

$$
\sup _{g \in G}\left|K^{D}(g)\right|=1
$$

Proof

Since $\left\|v^{D}\right\|=1$ and $J^{D}, T_{g}^{D}$ are isometric, $\left|K^{D}(g)\right| \leq 1$.

Now consider the family $\mathfrak{F} \equiv\left\{\zeta^{D}(g)=\left\langle T_{g}^{D} u, w\right\rangle\right\}$ of matrix elements. Here $D$ runs over $\Omega$, and $u, w$ are any unit vectors in $\mathcal{H}^{D}$. For two $\zeta^{D_{1}}(g), \zeta^{D_{2}}(g)$,

$$
\begin{aligned}
\overline{\zeta^{D}(g)} & =\zeta^{\bar{D}}(g), \\
\zeta^{D_{1}}(g)+\zeta^{D_{2}}(g) & =\zeta^{D_{1} \oplus D_{2}}(g), \\
\zeta^{D_{1}}(g) \times \zeta^{D_{2}}(g) & =\zeta^{D_{1} \otimes D_{2}}(g) .
\end{aligned}
$$

Therefore $\mathfrak{F}$ is a $*$-algebra contained in the $*$-algebra $\mathcal{C}^{b}(G)$ of all bounded continuous functions on $G$.

Define norm $\left\|\zeta^{D}\right\| \equiv \sup _{g \in G}\left|\zeta^{D}(g)\right|$ on $\mathfrak{F}$. Consider the completion $\mathfrak{F}^{C}$ of $\mathfrak{F}$ with respect to this norm. Then as a set of uniform limits of continuous functions of $\mathfrak{F}, \mathfrak{F}^{C}$ becomes a $C^{*}$-algebra of continuous functions on $G$.

Applying the Gelfand representation theorem, there exists a locally compact space $X$, and $\mathfrak{F}^{C}$ is isomorphic to the space $\mathcal{C}^{b}(X)$ of all bounded continuous functions on $X$ under the correspondence $\mathfrak{F}^{C} \ni f \longrightarrow f^{\sim} \in \mathcal{C}^{b}(X)$. A point $x$ of $X$ is considered as a homomorphic map such that

$$
\begin{aligned}
& \psi^{x}: \mathcal{C}^{b}(X) \longrightarrow \mathbf{C}, \\
& \psi^{x}(\varphi) \equiv \varphi(x) \quad\left(\varphi \in \mathcal{C}^{b}(X)\right) .
\end{aligned}
$$

For any element $g$ in $G$ and $f$ in $\mathfrak{F}^{C}$,

$$
f \longmapsto f(g)
$$

gives a homomorphic map from $\mathfrak{F}^{C}$ to $\mathbf{C}$. So there exits a unique element $x_{g}$ in $X$ as

$$
f(g)=f^{\sim}\left(x_{g}\right) .
$$

The existence of an SSUR assures us that the map $g \longmapsto x_{g}$ is one-to-one. So by this map, $G$ is embedded into $X$. But $\mathcal{C}^{b}(X)$ is given as the space of $\left\{f^{\sim} \mid f \in \mathfrak{F}^{C}\right\}$ and $\mathfrak{F}^{C} \subset \mathcal{C}^{b}(G)$. From this we conclude that the image of $G$ is dense in $X$. That is, for any $x \in X, \delta>0$, and $f^{\sim}(x) \in \mathfrak{F}^{C}$, there exists $g_{0} \in G$ such that

$$
\left|f^{\sim}\left(g_{0}\right)-f^{\sim}(x)\right|<\delta
$$


For a given isobirepresentation $\mathbf{J} \equiv\left\{J^{D}\right\}_{D \in \Omega}$, consider the map

$$
\zeta^{D}(g)=\left\langle T_{g}^{D} v^{D}, u^{D}\right\rangle \longrightarrow\left\langle T_{g}^{D} J^{D} v^{D}, u^{D}\right\rangle \equiv \theta_{\mathbf{J}}\left(\zeta^{D}\right)(g)
$$

By analogous consideration as in (2.5)-(2.7), we get

$$
\begin{aligned}
\overline{\theta_{\mathbf{J}} \zeta^{D}(g)} & =\overline{\left\langle T_{g}^{D} J^{D} v^{D}, u^{D}\right\rangle}=\left\langle\overline{T_{g}^{D} J^{D}} v^{\bar{D}}, u^{\bar{D}}\right\rangle=\left\langle T_{g}^{\bar{D}} J^{\bar{D}} v^{\bar{D}}, u^{\bar{D}}\right\rangle \\
& =\theta_{\mathbf{J}}\left(\zeta^{\bar{D}}\right)(g), \\
\theta_{\mathbf{J}}\left(\zeta^{D_{1}}\right)(g)+\theta_{\mathbf{J}}\left(\zeta^{D_{2}}\right)(g) & =\left\langle T_{g}^{D_{1}} J^{D_{1}} v^{D_{1}}, u^{D_{1}}\right\rangle+\left\langle T_{g}^{D_{2}} J^{D_{2}} v^{D_{2}}, u^{D_{2}}\right\rangle \\
& =\left\langle\left(T_{g}^{D_{1}} J^{D_{1}} v^{D_{1}} \oplus T_{g}^{D_{2}} J^{D_{2}} v^{D_{2}}\right),\left(u^{D_{1}} \oplus u^{D_{2}}\right)\right\rangle \\
& =\theta_{\mathbf{J}}\left(\zeta^{D_{1} \oplus D_{2}}\right)(g), \\
\theta_{\mathbf{J}}\left(\zeta^{D_{1}}\right)(g) \times \theta_{\mathbf{J}}\left(\zeta^{D_{2}}\right)(g) & =\left\langle T_{g}^{D_{1}} J^{D_{1}} v^{D_{1}}, u^{D_{1}}\right\rangle \times\left\langle T_{g}^{D_{2}} J^{D_{2}} v^{D_{2}}, u^{D_{2}}\right\rangle \\
& =\left\langle\left(T_{g}^{D_{1}} J^{D_{1}} v^{D_{1}} \otimes T_{g}^{D_{2}} J^{D_{2}} v^{D_{2}}\right),\left(u^{D_{1}} \otimes u^{D_{2}}\right)\right\rangle \\
& =\theta_{\mathbf{J}}\left(\zeta^{D_{1} \otimes D_{2}}\right)(g) .
\end{aligned}
$$

Consider the case $\Sigma_{j}\left\langle T_{g}^{D_{j}} v^{D_{j}}, u^{D_{j}}\right\rangle \equiv 0$ as a function on $G$ for some countable set $\left\{D_{j}\right\} \subset \Omega$ and $\left\{v^{D_{j}}, u^{D_{j}} \in \mathcal{H}^{D_{j}}\right\}$ such that $\Sigma_{j}\left\|v^{D_{j}}\right\|^{2}, \Sigma_{j}\left\|u^{D_{j}}\right\|^{2}<\infty$.

Put $D \equiv \Sigma_{j}^{\oplus} D_{j}, v \equiv \Sigma_{j}^{\oplus} v^{D_{j}}, u \equiv \Sigma_{j}^{\oplus} u^{D_{j}}$; then

for any $g, h \in G, \quad\left\langle T_{g}^{D} v, T_{h}^{D} u\right\rangle \equiv 0$,

that is, $\left[\left\{T_{g}^{D} v \mid g \in G\right\}\right] \perp\left[\left\{T_{h}^{D} u \mid h \in G\right\}\right]$.

The condition (B-2) shows that the operator $J^{D}$ of isobirepresentation $\mathbf{J} \equiv$ $\left\{J^{D}\right\}_{D \in \Omega}$ keeps invariant subspaces; therefore $\left[\left\{T_{g}^{D} v \mid g \in G\right\}\right] \perp J^{D} u$. This concludes

$$
0=\left\langle T_{g}^{D} v, J^{D} u\right\rangle=\Sigma_{j}\left\langle T_{g}^{D_{j}} v^{D_{j}}, J^{D_{j}} u^{D_{j}}\right\rangle .
$$

Therefore the map (2.13) generates a *algebra homomorphism

$$
f^{\sim}(g) \longrightarrow \theta_{\mathbf{J}}\left(f^{\sim}\right)(e) \equiv f^{\sim}\left(x_{J}\right)
$$

of the space $\mathfrak{F}$, and of $\mathfrak{F}^{C}$ to $C$; that is, it gives an element $x_{J} \in X$.

Put $f^{\sim}(g) \equiv\left\langle T_{g}^{D} v^{D}, J^{D} v^{D}\right\rangle$, and apply (2.12). We obtain

$$
\begin{aligned}
\left|f^{\sim}\left(g_{0}\right)-f^{\sim}\left(x_{J}\right)\right| & =\left|\left\langle T_{g_{0}}^{D} v^{D}, J^{D} v^{D}\right\rangle-\left\langle J^{D} v^{D}, J^{D} v^{D}\right\rangle\right| \\
& =\left|\left\langle T_{g_{0}}^{D} v^{D}, J^{D} v^{D}\right\rangle-1\right|=\left|1-K\left(g_{0}^{-1}\right)\right|<\delta .
\end{aligned}
$$

This proves (2.4).

In $[7$, Sections 7,8$]$, we give the following conditions to a topological group $G$ :

(W-3) For any cyclic unitary representation

$$
D \equiv\left\{\mathcal{H}^{D}, T_{g}^{D}, v^{D}\right\} \quad\left(\left\|v^{D}\right\|=1\right)
$$

and any birepresentation $\mathbf{U} \equiv\left\{U^{D}\right\}_{D}$, there holds

$$
\sup _{g \in G}\left|\left\langle T_{g}^{D} U^{D} v^{D}, v^{D}\right\rangle\right|=1
$$


$\left(\mathrm{W}-3^{\prime}\right)$ For any cyclic unitary representation

$$
D \equiv\left\{\mathcal{H}^{D}, T_{g}^{D}, v^{D}\right\} \quad\left(\left\|v^{D}\right\|=1\right),
$$

and any isobirepresentation $\mathbf{J} \equiv\left\{J^{D}\right\}_{D}$, there holds

$$
\sup _{g \in G}\left|\left\langle T_{g}^{D} J^{D} v^{D}, v^{D}\right\rangle\right|=1 .
$$

After the above argument, we obtain the following lemma.

\section{LEMMA 2.3}

If a topological group $G$ has an SSUR, then ( $W$-3') holds for $G$, and so does $(W-3)$.

We defined well-behaved group as a topological group which satisfies the conditions (T-1), (T-2) ((W-1), (W-2) in [8]), and (W-3), and we defined strongly well-behaved group as a topological group which satisfies the conditions (T-1), $\left(\mathrm{T}-2^{\prime}\right)\left((\mathrm{W}-1),\left(\mathrm{W}-2^{\prime}\right)\right.$ in $\left.[7]\right)$, and $\left(\mathrm{W}-3^{\prime}\right)$. Therefore we ge the following.

\section{COROLLARY 2.3.1}

(1) A T-type group is a well-behaved group.

(2) A strongly T-type group is a strongly well-behaved group.

As in Section 2, for representations of type $\left(D_{p}\right)$, the set $\left\{\left(D_{p}\right)\right\}_{D}$ gives an SSUR of $G$, and also

$$
\inf _{g \in G}\left(1-K^{D_{p}}(g)\right)=0 .
$$

We denote by $\Omega_{+}$the set of all cyclic representations $D=\left(\mathcal{H}^{D}, T_{g}^{D}, v^{D}\right)$ $\left(\left\|v^{D}\right\|=1\right)$ satisfying

$$
K^{D}(g)=\left\langle T_{g}^{D} J^{D} v^{D}, v^{D}\right\rangle \geq 0 \quad(g \in G) .
$$

As was shown, $\Omega_{+}$contains cyclic representations of type $\left(D_{p}\right)$.

Introduce a subset of $G$,

$$
F(D, \varepsilon) \equiv\left\{g \mid 1-K^{D}\left(g^{-1}\right)<\varepsilon\right\}
$$

for $\varepsilon>0, D \in \Omega_{+}$, and consider the family of subsets

$$
\mathrm{Z} \equiv\{F(D, \varepsilon)\}_{D \in \Omega_{+}, \varepsilon>0} .
$$

\section{LEMMA 2.4}

Let $G$ be a topological group with SSUR, and let $\mathbf{J}$ be an isobirepresentation of $G$. Then $\mathrm{Z}$ gives a Cauchy filter base on $G$ by the order of sets inclusion.

If a given $\mathbf{J}$ is a birepresentation $\mathbf{U} \equiv\left\{U^{D}\right\}_{D}$, that is, for any $D, J^{D}\left(=U^{D}\right)$ is a unitary operator, then $\mathrm{Z}$ is b-Cauchy. 
Proof

The arguments are almost the same as in $[8$, Sections 7,8$]$. So we will trace the argument there shortly.

Lemma 2.2 shows that for any $D \in \Omega_{+}$and $\varepsilon>0, F(D, \varepsilon)$ is not empty:

$$
\varepsilon_{1}>\varepsilon_{2} \quad \Longrightarrow \quad F\left(D, \varepsilon_{1}\right) \supseteq F\left(D, \varepsilon_{2}\right) .
$$

And for $D^{0} \equiv\left(D^{1} \otimes D^{2}\right)$, we obtain

$$
1-K^{D^{0}}\left(g^{-1}\right) \geq 1-K^{D^{1}}\left(g^{-1}\right), 1-K^{D^{2}}\left(g^{-1}\right) .
$$

Therefore

$$
F\left(D^{1}, \varepsilon\right) \cap F\left(D^{2}, \varepsilon\right) \supseteq F\left(D^{0}, \varepsilon\right) \neq \phi .
$$

This shows that $\mathrm{Z}$ is a filter base.

To show that $\mathrm{Z}$ is $l$-Cauchy, we showed in $[8,(7.8)]$ that the condition $1-$ $K^{D}\left(g^{-1}\right)<\varepsilon$ leads to

$$
\left\|U^{D} v^{D}-T_{g}^{D} v^{D}\right\| \leq(2 \varepsilon)^{1 / 2} .
$$

Using this relation we get for any $g, h \in F(D, \varepsilon)$,

$$
\left\|T_{g}^{D} v^{D}-T_{h}^{D} v^{D}\right\|=\left\|T_{h^{-1} g}^{D} v^{D}-v^{D}\right\| \leq 2(2 \varepsilon)^{1 / 2} .
$$

For an arbitrary given neighborhood $V$ of $e$ in $G$, if we take the above $D$ in $\Omega_{+}$as

$$
\left\{g \in G||\left\langle T_{g}^{D} v^{D}-v^{D}, v^{D}\right\rangle \mid<\delta\right\} \subset V
$$

then $4 \varepsilon<\delta$ leads to

$$
F(D, \varepsilon)^{-1} F(D, \varepsilon) \subset V,
$$

that is, $\mathrm{Z}$ is $l$-Cauchy.

Next we consider the case where a given isobirepresentation $\mathbf{J}$ is a birepresentation $\mathbf{U}=\left\{U^{D}\right\}_{D}$.

We will show that the family of subsets

$$
\mathrm{Z}^{-1} \equiv\left\{F(D, \varepsilon)^{-1}\right\}_{D \in \Omega_{+}, \varepsilon>0}
$$

gives a Cauchy filter base. In the above arguments, we proved that $\mathrm{Z} \equiv\{F(D, \varepsilon)\}$ gives a filter base, so $\mathrm{Z}^{-1} \equiv\left\{F(D, \varepsilon)^{-1}\right\}$ is also a filter base. Hence it is sufficient to see that $\mathrm{Z}^{-1}$ is Cauchy.

For an arbitrary given neighborhood $V_{0}$ of $e$ in $G$ and $\delta>0$, take $D \in \Omega_{+}$ and a normalized vector $w$ in $\mathcal{H}^{D}$ as

$$
\left\{g \in G||\left\langle T_{g}^{D} w-w, w\right\rangle \mid<\delta\right\} \subset V_{0} .
$$

Since $U^{D}$ is unitary, we can take $v^{D} \equiv\left(U^{D}\right)^{-1} w$ and $\varepsilon>0$ as $\varepsilon<\delta$. above.

Consider $K^{D}(g)=\left\langle T_{g}^{D} U^{D} v^{D}, v^{D}\right\rangle \geq 0(g \in G)$, and consider $F(D, \varepsilon)$ as 
As in (2.23), the relation $1-K^{D}\left(g^{-1}\right)<\varepsilon$ gives that for any $g \in F(D, \varepsilon)$,

$$
\begin{aligned}
\left\|T_{g^{-1}}^{D} w-\left(U^{D}\right)^{-1} w\right\| & =\left\|w-T_{g}^{D}\left(U^{D}\right)^{-1} w\right\| \\
& =\left\|U^{D} v^{D}-T_{g} v^{D}\right\| \leq(2 \varepsilon)^{1 / 2} .
\end{aligned}
$$

Consequently we see that for any $g, h \in F(D, \varepsilon)$,

$$
\begin{aligned}
\left\|T_{h g^{-1}}^{D} w-w\right\| & =\left\|T_{g^{-1}}^{D} w-T_{h^{-1}}^{D} w\right\| \\
& \leq\left\|T_{g^{-1}}^{D} w-\left(U^{D}\right)^{-1} w\right\|+\left\|\left(U^{D}\right)^{-1} w-T_{h^{-1}}^{D} w\right\| \leq 2(2 \varepsilon)^{1 / 2} .
\end{aligned}
$$

By analogous arguments as after $(2.24)$ or in the proof of $[8$, Section 7 , Lemma 7.4], this leads to $h g^{-1} \in V_{0}$. Therefore

$$
F(D, \varepsilon) F(D, \varepsilon)^{-1} \subset V_{0} .
$$

This shows that $\left\{F(D, \varepsilon)^{-1}\right\}$ gives a Cauchy filter, and $\mathrm{Z}$ is b-Cauchy.

\section{Proof of a Tannaka-type weak duality theorem for T-type groups and strongly T-type groups}

PROPOSITION 3.1

(1) For a T-type group $G$, a weak Tannaka-type u-duality theorem is valid.

(2) For a strongly-T-type group G, a weak Tannaka-type i-duality theorem is valid.

Proof

We fix an isobirepresentation $\mathbf{J} \equiv\left\{J^{D}\right\}$ and show that there exists a unique $g$ in $G$ such that

$$
\left\{J^{D}\right\}=\left\{T_{g}^{D}\right\} .
$$

Lemma 2.4 shows that for T-type (also for strongly T-type) group $G$ and $\mathbf{J}$ as above, $\mathrm{Z} \equiv\{F(D, \varepsilon)\}_{D \in \Omega_{+}, \varepsilon>0}$ gives a Cauchy filter base.

Especially in the case that $\mathbf{J}$ is a birepresentation, $\mathrm{Z}$ give a $b$-Cauchy filter base.

Therefore if $G$ is T-type and $\mathbf{J}$ is a birepresentation, or $G$ is strongly-T-type and $\mathbf{J}$ is an isobirepresentation, $\mathrm{Z}$ converges to a point in $G$.

In both cases we write this limit point as $\left(g_{\mathbf{J}}\right)^{-1}$. Then,

$$
\bigcap_{(D, \varepsilon)} \overline{F(D, \varepsilon)}=\left\{\left(g_{\mathbf{J}}\right)^{-1}\right\} \text {. }
$$

So $1=\left\langle T_{\left(g_{\mathbf{J}}\right)^{-1}}^{D} J^{D} v^{D}, v^{D}\right\rangle$, and

$$
\forall D \in \Omega_{+}, \quad J^{D} v^{D}=T_{g_{\mathbf{J}}}^{D} v^{D} .
$$

For a general cyclic representation $D$, consider $\left(D_{p}\right) \in \Omega_{+}$as in Section 1 ; then we obtain from $J^{D_{p}} v_{p}=T_{g_{\mathbf{J}}}^{D_{p}} v_{p}$,

$$
I w_{0} \oplus J^{D} w \oplus J^{\bar{D}} \bar{w}=I w_{0} \oplus T_{g_{\mathbf{J}}}^{D} w \oplus T_{g_{\mathbf{J}}}^{\bar{D}} \bar{w} .
$$


So for any $D$ in $\Omega$, we get $J^{D} w=T_{g_{\mathbf{J}}}^{D} w$.

This concludes the proof of the assertion.

\section{Converse of duality theorems for T-type or strongly T-type groups}

PROPOSITION 4.1

For a $T_{2}$-topological group $G$, if a weak Tannaka-type u-duality theorem holds, then $G$ has an SSUR; that is, the condition (T-1) in Definition 1.3 is satisfied.

Proof

By Lemma 1.1, the inverse map of (1.5) must be continuous. A fundamental system of neighborhoods $V$ of $e$ in the image $G_{J}$ of $G$ in $\mathcal{U}$ is given as the collection of

$$
V_{1} \equiv \bigcap_{1 \leq j \leq n}\left\{\mathbf{T}_{g}=\left(T_{g}^{D}\right)_{D \in \Omega} \mid\left\|v_{j}-T_{g}^{D_{j}} v_{j}\right\|^{2}<\varepsilon_{j}\right\}
$$

for a finite set $\left\{\left(D_{j}, v_{j}, \varepsilon_{j}\right)\right\}$, where $D_{j} \in \Omega$ and $v_{j} \in \mathcal{H}^{D_{j}}\left(\left\|v_{j}\right\|=1\right), \varepsilon_{j}>0$ $(j=1,2, \ldots, n)$.

Consider the representations $D_{0} \equiv \Sigma_{j}^{\oplus} D_{j}$ and $v_{0}=n^{-(1 / 2)} \Sigma_{j}^{\oplus} v_{j}, \quad \varepsilon_{0}=$ $\operatorname{Min}_{j} \varepsilon_{j}$; then

$$
V_{1} \supseteq V_{2}\left(\varepsilon_{0}\right) \equiv\left\{\mathbf{T}_{g}=\left(T_{g}^{D}\right)_{D \in \Omega} \mid\left\|v_{0}-T_{g}^{D_{0}} v_{0}\right\|^{2}<\varepsilon_{0}\right\} .
$$

The evaluation

$$
\begin{aligned}
\left\|v_{0}-T_{g}^{D_{0}} v_{0}\right\|^{2} & =2\left(1-\Re\left(\left\langle T_{g}^{D_{0}} v_{0}, v_{0}\right\rangle\right)\right) \\
& \leq 2\left|1-\left\langle T_{g}^{D_{0}} v_{0}, v_{0}\right\rangle\right|
\end{aligned}
$$

shows that, if we take $\delta<2^{-1} \varepsilon_{0}$, then

$$
V_{2}\left(\varepsilon_{0}\right) \supset V_{\delta} \equiv\left\{\mathbf{T}_{g}=\left(T_{g}^{D}\right)_{D \in \Omega}|| 1-\left\langle T_{g}^{D_{0}} v_{0}, v_{0}\right\rangle \mid<\delta\right\} .
$$

Since the inverse map of (1.5) is continuous, for any neighborhood $V$ of $e$ in $G$, there exist $V, V_{1}, V_{2}\left(\varepsilon_{0}\right)$, and $V_{\delta}$ such that

$$
V \supseteq V_{1} \supseteq V_{2}\left(\varepsilon_{0}\right) \supseteq V_{\delta} .
$$

This shows the separating condition (T-1) in Definition 1.3.

As is shown in Proposition 0.3, u-duality follows from i-duality. Then we have the following.

\section{COROLLARY 4.1.1}

For a topological group $G$, if the weak Tannaka-type $i$-duality theorem holds, then the condition (T-1) is satisfied.

Now we discuss about the conditions (T-2) and $\left(\mathrm{T}-2^{\prime}\right)$. 
PROPOSITION 4.2

Let $G$ be a $T_{2}$-topological group.

(1) For the weak Tannaka-type i-duality theorem to hold, G must be complete; that is, the condition $\left(T-2^{\prime}\right)$ should hold.

(2) For the weak Tannaka-type u-duality theorem to hold, $G$ must be bcomplete; that is, the condition (T-2) should hold.

Proof

The map (1.6) is isomorphic. We consider the image $G_{J}$ in $\mathbf{U}(\Omega) \subset \mathbf{J}(\Omega)$.

Lemma 1.3 asserts that a Cauchy filter base $\mathcal{F}_{J}$ on $G_{J}$ has a limit $\mathbf{B} \equiv\left(B^{D}\right)_{D}$ in $\mathbf{J}(\Omega)$. And if $\mathcal{F}$ is a $b$-Cauchy filter base, then $\mathbf{B}$ is in $\mathbf{U}(\Omega)$.

Obviously any element of $G_{J}$ satisfies the conditions (B-1)-(B-4) of birepresentation and isobirepresentation in Section 0. And these conditions are valid for its limit point $\mathbf{B} \equiv\left(B^{D}\right)_{D}$ of these elements too.

Consequently, we see that $\mathbf{B}$ is an isobirepresentation. If $\mathbf{B}$ is in $\mathbf{U}(\Omega)$, it is a birepresentation too.

If the weak Tannaka-type i-duality theorem holds for $G$, then $\mathbf{B} \in G_{J}$. This shows that any Cauchy filter base $\mathcal{F}$ on $G_{J}$ converges to a point in $G_{J}$; that is, $G_{J}$ is complete. Equivalently $G$ must be complete; that is, $G$ is strongly T-type.

In the case where the weak Tannaka-type u-duality theorem holds for $G$, a $b$-Cauchy filter base $\mathcal{F}$ must converge to a point $\mathbf{B}$ in $\mathcal{U}(\Omega)$. That is, $\mathbf{B}$ turns to be a birepresentation. So the assumption that the weak Tannaka-type u-duality is valid for $G$ induces the assertion that $G$ is $b$-complete. Hence, in this case, $G$ is a T-type group.

\section{Case of locally compact groups and NOS-groups}

We consider the case of locally compact groups. Here we quote the papers [3]-[5], and $[8]$.

In [3] and [4], we have shown that, for locally compact groups, the b-duality theorem is valid. And in [5] and [8], it is shown that b-duality is false if the group is not locally compact.

Altogether we showed

$$
\text { b-duality } \Longleftrightarrow \text { locally compact. }
$$

And it is remarkable that for locally compact groups to prove the weak duality theorem we do not need the condition (B-4) in the definition of birepresentations (cf. [3], [4]).

Next we consider the case where c-duality is false for a locally compact group $G$.

In [4], we introduced so-called Katz-Takesaki operator $W_{\mathfrak{R}}$ on the regular representation $\mathfrak{R} \equiv\left(\mathfrak{H}, R_{g}\right), \mathfrak{H}=L^{2}(G)$ of a given locally compact group $G$. $W_{\mathfrak{R}}$ is a unitary operator defined on the space $\mathfrak{H} \otimes \mathfrak{H}$. And $W_{\mathfrak{R}}$ gives an intertwining operator from $\mathfrak{R} \otimes \mathfrak{R}$ to $\Sigma^{\oplus} \mathfrak{R}$ (a multiple of $\mathfrak{R}$ ). $W_{\mathfrak{R}}$ is written for any fixed 
$\operatorname{CONS}\left\{\Phi_{\alpha}\right\}_{\alpha}$ in $\mathfrak{H}$ as

$$
\left(W_{\mathfrak{R}}\left(f_{1} \otimes f_{2}\right)\right)(g)=\left\{\left\langle R_{g} f_{2}, \Phi_{\alpha}\right\rangle f_{1}(g)\right\}_{\alpha} \in \Sigma_{\alpha}^{\oplus} \mathfrak{H} \quad\left(f_{1} \otimes f_{2} \in \mathfrak{H} \otimes \mathfrak{H}\right)
$$

(cf. [4]).

The group $G$ satisfies the b-duality theorem, so $G$ is identified as

$$
G \approx G_{R} \equiv\left\{U \mid W_{\mathfrak{R}}(U \otimes U)=(I \otimes U) W_{\mathfrak{R}} \neq 0\right\} \subset U(\mathfrak{H}) .
$$

To extend the discussion to the case of c-duality, we must extend $G_{R}$ to the space

$$
G_{C}^{R} \equiv\left\{C \mid W_{\mathfrak{R}}(C \otimes C)=(I \otimes C) W_{\mathfrak{R}} \neq 0\right\} \subset C(\mathfrak{H}) .
$$

Here $C(\mathfrak{H})$ is the space of closed operators $C$ on $\mathfrak{H}$ with a common domain $\mathcal{D}_{R}$ satisfying (B-041)-(B-044) for the case

$$
D_{1}=D_{2}=\mathfrak{R}
$$

An element $C$ of $G_{C}^{R}$ is called an admissible operator. It is a closed operator with domain $\mathcal{D}_{R}$ and range in the same $\mathcal{D}_{R}$ satisfying

$$
W_{\mathfrak{R}}(C \otimes C)=(I \otimes C) W_{\mathfrak{R}} .
$$

We must show the following proposition which is an extension of $[3$, Lemma 2.4].

\section{PROPOSITION 5.1}

For any cl-birepresentation $\mathbf{C}=\left\{C^{D}\right\}_{D \in \Omega}$, its component $C^{\mathfrak{R}}$ on the regular representation $\mathfrak{R}$ is an admissible operator. For two cl-birepresentations $\mathbf{C}_{1}$ and $\mathbf{C}_{2}$, if

$$
C_{1}^{\mathfrak{R}}=C_{2}^{\mathfrak{R}},
$$

then $\mathbf{C}_{1}=\mathbf{C}_{2}$, that is, the same $C_{1}^{D}=C_{2}^{D}$ for any $D$ in $\Omega$.

\section{Proof}

The proof is done similarly to the argument in [3]. It was shown that $D \otimes \Re$ is unitary equivalent to $\Sigma_{\operatorname{dim} D}^{\oplus} \mathfrak{R}$ with an intertwining operator $W_{D}$. So, for a cl-birepresentation $\mathbf{C}=\left\{C^{D}\right\}$,

$$
W_{D}\left(C^{D} \otimes C^{\mathfrak{R}}\right)=\left(I \otimes C^{\mathfrak{R}}\right) W_{D} .
$$

When $D=\mathfrak{R}$, this shows that the operator $C^{\mathfrak{R}}$ is an admissible operator. For two $\mathbf{C}_{i} \equiv\left(C_{i}^{D}\right)_{D \in \Omega}(i=1,2)$,

$$
W_{D}\left(C_{1}^{D} \otimes C^{\mathfrak{R}}\right)=\left(I \otimes C^{\mathfrak{R}}\right) W_{D}=W_{D}\left(C_{2}^{D} \otimes C^{\mathfrak{R}}\right) ;
$$

that is,

$$
C_{1}^{D} u \otimes C^{\Re} v=C_{2}^{D} u \otimes C^{\Re} v
$$

for any $u$ in $\mathcal{D}^{D}$ and $v$ in $\mathcal{D}^{\Re}$. The nonzero assumption of cl-birepresentation leads $C_{1}^{D} u=C_{2}^{D} u$ for any $u$ in $\mathcal{D}^{D}$, that is, 


$$
C_{1}^{D}=C_{2}^{D}
$$

for any $D$ in $\Omega$.

After Proposition 5.1, to show c-duality, it is sufficient to show that for any admissible operator $C$, there exists an element $g$ in $G$ such that $C=R_{g}$.

\section{PROPOSITION 5.2}

Let $C_{1}$ and $C_{2}$ be two admissible operators; then their product $C_{1} C_{2}$ is also an admissible operator.

Proof

$$
\begin{aligned}
W_{R}\left(C_{1} C_{2} \otimes C_{1} C_{2}\right) & =W_{\mathfrak{R}}\left(C_{1} \otimes C_{1}\right)\left(C_{2} \otimes C_{2}\right)=\left(I \otimes C_{1}\right) W_{\mathfrak{R}}\left(C_{2} \otimes C_{2}\right) \\
& =\left(I \otimes C_{1}\right)\left(I \otimes C_{2}\right) W_{\mathfrak{R}}=\left(I \otimes C_{1} C_{2}\right) W_{\mathfrak{R}} .
\end{aligned}
$$

PROPOSITION 5.3

For an admissible operator $C$, its conjugate operator $\bar{C}$ is an admissible operator too.

Proof

We have

$$
\begin{aligned}
W_{\mathfrak{R}}(\bar{C} \otimes \bar{C}) & =\overline{(C \otimes C) \overline{W_{\mathfrak{R}}}}=\overline{(C \otimes C) W_{\mathfrak{R}}^{-1}} \\
& =\overline{W_{\mathfrak{R}}^{-1}(I \otimes C)}=\overline{\overline{W_{\mathfrak{R}}}(I \otimes C)}=(I \otimes \bar{C}) W_{\mathfrak{R}} .
\end{aligned}
$$

\section{COROLLARY 5.3.1}

For an admissible operator $C$, the positive definite operator $\bar{C} C$ (i.e., $C_{P}$ ) is an admissible operator too; that is,

$$
W_{\mathfrak{R}}\left(C_{P} \otimes C_{P}\right)=\left(I \otimes C_{P}\right) W_{\mathfrak{R}} .
$$

Proof

From Propositions 5.2 and 5.3, the result is direct.

COROLLARY 5.3.2

For an admissible operator $C$, the positive definite operator $\left(C_{P}\right)^{t} \equiv(\bar{C} C)^{t} \quad(t \in$ R) is an admissible operator too.

Proof

For positive definite operators $C_{P}$, we can define uniquely $t$-power $\left(C_{P}\right)^{t}$ :

$$
\begin{aligned}
\left(C_{P}\right)^{t} \otimes\left(C_{P}\right)^{t} & =\left(C_{P} \otimes C_{P}\right)^{t}=\left(W_{\mathfrak{R}}^{-1}\left(I \otimes C_{P}\right) W_{\mathfrak{R}}\right)^{t} \\
& =W_{\mathfrak{R}}^{-1}\left(I \otimes C_{P}\right)^{t} W_{\mathfrak{R}}=W_{\mathfrak{R}}^{-1}\left(I \otimes\left(C_{P}\right)^{t}\right) W_{\mathfrak{R}} .
\end{aligned}
$$

This shows that $\left(C_{P}\right)^{t}$ is an admissible operator. 
PROPOSITION 5.4

Take the spectral decomposition

$$
\left(C_{P}\right)^{t}=\int_{0}^{\infty} \lambda^{t} d E(\lambda)
$$

in Corollary 5.3.2, and consider the self-adjoint operator

$$
L\left(\equiv \log C_{P}\right) \equiv \int_{0}^{\infty}(\log \lambda) d E(\lambda) ;
$$

then

$$
W_{\mathfrak{R}}(L \otimes I+I \otimes L)=(I \otimes L) W_{\mathfrak{R}} .
$$

Proof

For $v$ and $u$ in $\mathcal{D}_{R}$, take derivatives with respect to $t$ of the both sides of (5.11) in the strong sense, and put $t=0$ as shown below:

$$
\begin{aligned}
\left(\int_{0}^{\infty} \lambda^{t} d E(\lambda) \otimes \int_{0}^{\infty} \lambda^{t} d E(\lambda)\right)(u \otimes v) & =\left(C_{P}\right)^{t} \otimes\left(C_{P}\right)^{t}(u \otimes v) \\
& =W_{\mathfrak{R}}^{-1}\left(I \otimes\left(C_{P}\right)^{t}\right) W_{\mathfrak{R}}(u \otimes v) \\
& =W_{\mathfrak{R}}^{-1}\left(I \otimes \int_{0}^{\infty} \lambda^{t} d E(\lambda)\right) W_{\mathfrak{R}}(u \otimes v) .
\end{aligned}
$$

Then

$$
\begin{aligned}
\left.(d / d t)\left(C_{P}\right)^{t} u\right|_{t=0} & =\int_{0}^{\infty} \log (\lambda) d E(\lambda) u \equiv L u, \\
\left.(d / d t)\left(C_{P}\right)^{t}(u \otimes v)\right|_{t=0} & =(L u) \otimes v+u \otimes(L v) \\
& =(L \otimes I+I \otimes L)(u \otimes v),
\end{aligned}
$$

and combining these results, we get

$$
W_{\mathfrak{R}}(L \otimes I+I \otimes L)(u \otimes v)=(I \otimes L) W_{\mathfrak{R}}(u \otimes v) .
$$

\section{PROPOSITION 5.5}

For a nonunitary admissible operator $C$, there exists a one-parameter subgroup $\left\{\left(C_{P}\right)^{i t}(t \in \mathbf{R})\right\}$ of admissible unitary operators.

Proof

Since the operator $C$ is not unitary, the above $C_{P}$ is not $I$, so $L$ is not zero, $\left\{\left(C_{P}\right)^{i t}=\exp (i t L)(t \in \mathbf{R})\right\}$ is a group of unitary operators, and

$$
\begin{aligned}
\left(C_{P}\right)^{i t} \otimes\left(C_{P}\right)^{i t} & =\exp (i t L) \otimes \exp (i t L) \\
& =\exp (i t(L \otimes I+I \otimes L)) \\
& =W_{\mathfrak{R}}^{-1} \exp (i t(I \otimes L)) W_{\mathfrak{R}} \\
& =W_{\mathfrak{R}}^{-1}\left(I \otimes\left(C_{P}\right)^{i t}\right) W_{\mathfrak{R}} .
\end{aligned}
$$

Therefore $\left(C_{P}\right)^{i t}$ is an admissible operator. 
PROPOSITION 5.6

A locally compact NOS-group $G$ satisfies c-duality.

Proof

If c-duality is not valid for $G$, there exists a nontrivial cl-birepresentation $\mathbf{C} \equiv$ $\left(C^{D}\right)_{D \in \Omega}$ of $G$, which is not a birepresentation. Let $C^{\mathfrak{R}}$ be its component on the regular representation $\mathfrak{R}$.

By Proposition 5.1, $C^{\mathfrak{R}}$ is nonzero. If $C^{\mathfrak{R}}$ is unitary, the b-duality theorem for locally compact groups asserts that $C^{\mathfrak{R}}$ must be an element of $G_{R}$. So by Proposition 5.1, C must be a birepresentation.

Consequently, $C^{\mathfrak{R}}$ is nonzero and nonunitary.

Thus, using Proposition 5.5, we get a one-parameter subgroup of admissible unitary operators. Again by the b-duality theorem, $G$ must have a one-parameter subgroup. This contradicts our assumption that $G$ is an NOS-group.

Next we consider the case where the c-duality theorem is valid for a topological group $G$.

PROPOSITION 5.7

If $G$ has a one-parameter subgroup, then the c-duality theorem is false for $G$.

Proof

Let $K=\left\{g_{t}\right\}_{t}$ be a one-parameter subgroup of $G$. Then $\left\{T_{g_{t}}^{D}\right\}_{t}$ is a one-parameter subgroup in $\mathcal{U}\left(\mathcal{H}^{D}\right)$ for any $D$ in $\Omega$. Especially for the regular representation, $\left\{R_{g_{t}}\right\}_{t}$ is a one-parameter subgroup in $\mathcal{U}\left(L^{2}(G)\right)$.

For a $C^{\infty}$-function $f$ with compact support on $\mathbf{R}$, we define

$$
T_{f}^{D} \equiv \int f(t) T_{g_{t}}^{D} d t
$$

Then, for any $v^{D}$ in $\mathcal{H}^{D}, T_{f}^{D} v^{D}$ is a $C^{\infty}$-vector with respect to the parameter $t$.

By Stone's theorem, there exists a self-adjoint operator $A^{D}$ on the space $\mathcal{H}^{D}$, and $\left\{T_{f}^{D} v^{D} \mid v^{D} \in \mathcal{H}^{D}, f \in C^{\infty}(\mathbf{R})\right\}$ spans the domain of $A^{D}$ and

$$
T_{g_{t}}^{D}=\exp \left(i A^{D} t\right) \quad(\forall t \in \mathbf{R}) \quad=\int e^{i \lambda t} d E^{D}(\lambda) .
$$

Here

$$
A^{D}=\int \lambda d E^{D}(\lambda)
$$

is the spectral decomposition of $A$.

Since $T_{g_{t}}^{D}$ satisfies (B-1)-(B-3),

$$
\exp \left(i A^{D_{1}} t\right) \otimes \exp \left(i A^{D_{2}} t\right)=\exp \left(i A^{D_{1} \otimes D_{2}} t\right) .
$$

For the case where $D_{2}$ is a regular representation, 


$$
\exp \left(i A^{D_{1}} t\right) \otimes \exp \left(i A^{\Re} t\right)=W_{D_{1}}^{-1}\left(I \otimes \exp \left(i A^{\Re} t\right)\right) W_{D_{1}} .
$$

Here $W_{D_{1}}$ is the intertwining operator corresponding to the equivalence relation of

$$
D_{1} \otimes \Re \sim_{W_{D_{1}}} \Sigma_{\operatorname{dim}\left(D_{1}\right)}^{\oplus} \mathfrak{R} .
$$

Moreover, when $D_{1}$ is $\mathfrak{R}$,

$$
\exp \left(i A^{\mathfrak{R}} t\right) \otimes \exp \left(i A^{\Re} t\right)=W_{\mathfrak{R}}^{-1}\left(I \otimes \exp \left(i A^{\mathfrak{R}} t\right)\right) W_{\mathfrak{R}} .
$$

In the cases (5.19) and (5.20), we differentiate with respect to $t$ both sides and put $t=0$; then we obtain

$$
\begin{gathered}
A^{D_{1}} \otimes I_{\mathfrak{R}}+I_{D_{1}} \otimes A^{\mathfrak{R}}=W_{D_{1}}^{-1}\left(I_{D} \otimes A^{\mathfrak{R}}\right) W_{D_{1}}, \\
A^{\mathfrak{R}} \otimes I_{\mathfrak{R}}+I_{\mathfrak{R}} \otimes A^{\mathfrak{R}}=W_{\mathfrak{R}}^{-1}\left(I_{\mathfrak{R}} \otimes A^{\mathfrak{R}}\right) W_{\mathfrak{R}} .
\end{gathered}
$$

For the spectral decomposition (5.18) of $A^{D}$ and for any $a, b \in \mathbf{R}$, take the projection operator

$$
P_{(a, b)}^{D} \equiv \int_{a}^{b} d E^{D}(\lambda) .
$$

The subspace $\mathcal{D}_{0}^{D} \equiv \bigcup_{(a, b)} P_{(a, b)} \mathcal{H}^{D}$ is dense in $\mathcal{H}^{D}$ and contained in the domain of $A^{D}$.

Put for any $v \in \mathcal{D}_{0}^{D}$,

$$
C_{0}^{D} v \equiv \exp \left(A^{D}\right) v=\sum_{k=0}^{\infty}(k !)^{-1}\left(A^{D}\right)^{k} v .
$$

The right-hand side converges in the norm sense. $C_{0}^{D}$ is closable, and its closure $C^{D}$ is a positive definite operator.

Moreover, for any $v^{D} \in \mathcal{D}_{0}^{D}$ and $v^{\Re} \in \mathcal{D}_{0}^{\Re}$,

$$
\begin{aligned}
\left(C_{0}^{D} \otimes C^{\mathfrak{R}}\right)\left(v^{D} \otimes v^{\mathfrak{R}}\right) & \\
= & \left(C_{0}^{D} v^{D} \otimes C^{\mathfrak{R}} v^{\Re}\right) \\
& =\left(\sum_{k=0}^{\infty}(k !)^{-1}\left(A^{D}\right)^{k}\right) v^{D} \otimes\left(\sum_{k=0}^{\infty}(k !)^{-1}\left(A^{\mathfrak{R}}\right)^{k}\right) v^{\mathfrak{R}} \\
& =\sum_{k=0}^{\infty}(k !)^{-1}\left(A^{D} \otimes I_{\mathfrak{R}}+I_{D} \otimes A^{\mathfrak{R}}\right)^{k}\left(v^{D} \otimes v^{\mathfrak{R}}\right) \\
& =W_{D}^{-1}\left(\sum_{k=0}^{\infty}(k !)^{-1}\left(I_{D} \otimes A^{\mathfrak{R}}\right)^{k}\right) W_{D}\left(v^{D} \otimes v^{\mathfrak{R}}\right) \\
& =W_{D}^{-1}\left(I_{D} \otimes\left(\sum_{k=0}^{\infty}(k !)^{-1}\left(A^{\mathfrak{R}}\right)^{k}\right)\right) W_{D}\left(v^{D} \otimes v^{\mathfrak{R}}\right) \\
& =W_{D}^{-1}\left(I_{D} \otimes C^{\mathfrak{R}}\right) W_{D}\left(v^{D} \otimes v^{\mathfrak{R}}\right),
\end{aligned}
$$




$$
\begin{aligned}
& \left(C^{\mathfrak{R}} \otimes C^{\mathfrak{R}}\right)\left(v_{1}^{\mathfrak{R}} \otimes v_{2}^{\Re}\right) \\
& =\left(C^{\mathfrak{R}} v_{1}^{\mathfrak{R}} \otimes C^{\mathfrak{R}} v_{2}^{\mathfrak{R}}\right) \\
& =\left(\sum_{k=0}^{\infty}(k !)^{-1}\left(A^{\mathfrak{R}}\right)^{k}\right) \otimes\left(\sum_{k=0}^{\infty}(k !)^{-1}\left(A^{\mathfrak{R}}\right)^{k}\right)\left(v_{1}^{\mathfrak{R}} \otimes v_{2}^{\mathfrak{R}}\right) \\
& =\left(\sum_{k=0}^{\infty}(k !)^{-1}\left(A^{\mathfrak{R}} \otimes I_{\mathfrak{R}}+I_{R} \otimes A^{\mathfrak{R}}\right)^{k}\right)\left(v_{1}^{\mathfrak{R}} \otimes v_{2}^{\mathfrak{R}}\right) \\
& =W_{\mathfrak{R}}^{-1}\left(I_{R} \otimes\left(\sum_{k=0}^{\infty}(k !)^{-1}\left(A^{\mathfrak{R}}\right)^{k}\right)\right) W_{\mathfrak{R}}\left(v_{1}^{\mathfrak{R}} \otimes v_{2}^{\mathfrak{R}}\right) \\
& =W_{\mathfrak{R}}^{-1}\left(I_{R} \otimes C^{\mathfrak{R}}\right) W_{\mathfrak{R}}\left(v_{1}^{\mathfrak{R}} \otimes v_{2}^{\Re}\right) .
\end{aligned}
$$

This equality is valid on dense sets of domains of operators in both sides, so taking the closure of operators, we get that $C^{\mathfrak{R}}$ is a nonzero, nonunitary admissible operator, and $\mathbf{C} \equiv\left\{C^{D}\right\}$ gives a cl-birepresentation not belonging to $G_{J}$.

This shows that c-duality is false for $G$.

\section{Main theorem and examples}

Summarizing the results in Sections $3-5$, we obtain the following.

\section{MAIN THEOREM}

Let $G$ be a $T_{2}$-topological group.

(1) For $G$, the weak Tannaka-type u-duality theorem holds if and only if $G$ is a T-type group.

(2) For $G$, the weak Tannaka-type i-duality theorem holds if and only if $G$ is a strongly T-type group.

(3) For $G$, the weak Tannaka-type b-duality theorem holds if and only if $G$ is a locally compact group.

(4) For $G$, the weak Tannaka-type c-duality theorem holds if and only if $G$ is a locally compact NOS-group.

\section{EXAMPLE 1}

Let $\mathcal{H}$ be a Hilbert space of infinite dimension, and let $G \equiv U(\mathcal{H})$ be the group of all unitary operators on $\mathcal{H}$ with the weak (resp., strong) topology of operator space.

\section{LEMMA 6.1}

The group $G=U(\mathcal{H})$ is a topological group and has an SSUR. 
Proof

$G$ is a topological group by the strong topology, and this topology is equivalent to the weak one. Consider the identical representation $D_{0} \equiv\left\{\mathcal{H}, T_{U}\right\}$,

$$
G \ni U \longmapsto T_{U}(\equiv U) \in U(\mathcal{H}) .
$$

Evidently this representation is cyclic with cyclic vector $v$, which is any normalized vector in $\mathcal{H}$. We denote this cyclic representation as $D_{v} \equiv\left\{\mathcal{H}, T_{U}, v\right\}$ and take the family $\Omega_{0} \equiv\left\{D_{v}\right\}_{v}$. We show that this family gives an SSUR of $G$.

A fundamental system of neighborhoods of $e(=I)$ is given by the family of sets

$$
\mathcal{V} \equiv\{V(v, \varepsilon) \equiv\{U \in G \mid\|U v-v\|<\varepsilon\} \mid v \in \mathcal{H},\|v\|=1, \varepsilon>0\} .
$$

For any $D_{v}$ in $\Omega_{0},\|U v-v\|^{2}=2(1-\Re\langle U v, v\rangle)$ and $\left|1-\left\langle T_{U} v, v\right\rangle\right|^{2}=\mid 1-$ $\left.\Re\left(\left\langle T_{U} v, v\right\rangle\right)\right|^{2}+\left|\Im\left(\left\langle T_{U} v, v\right\rangle\right)\right|^{2}$, so

$$
\left|1-\left\langle T_{U} v, v\right\rangle\right| \geq\left|1-\Re\left\langle T_{U} v, v\right\rangle\right|=(1 / 2)\|U v-v\|^{2} .
$$

As in Section 1, we put $\eta(g) \equiv\left\langle T_{U} v, v\right\rangle$ and $F(D, \varepsilon) \equiv\{g \in G|| 1-\eta(g) \mid<\delta\}$.

This means that for $\delta \leq 2^{-1} \varepsilon^{2}$,

$$
F(D, \delta) \subset V(v, \varepsilon) ;
$$

that is, $\Omega_{0}$ gives an SSUR for $G$.

\section{LEMMA 6.2}

The group $G \equiv U(\mathcal{H})$ is b-complete but not complete.

\section{Proof}

Lemma 1.3(1) shows that any Cauchy filter base on $G_{J}$ converges to an element in $\mathcal{J}(\Omega)$, and Lemma 1.3(2) shows that any $b$-Cauchy filter base converges to an element in $\mathcal{U}(\Omega)$.

The map $G=U(\mathcal{H}) \ni U \longrightarrow U \in U(\mathcal{H})$ gives a unitary representation of $G$. As a component of $G_{J}, U(\mathcal{H})=G$ is $b$-complete.

In $\mathcal{H}$, we take a countable infinite orthonormal system $L \equiv\left\{v_{1}, v_{2}, \ldots\right\}$ and the closed subspace $\mathcal{H}_{0}$ spanned by $L$. We consider the unitary operator $U_{n}$ which is identity on $\left(\mathcal{H}_{0}\right)^{\perp}$ and is defined on $\mathcal{H}_{0}$ as follows:

$$
U_{n}\left(\sum_{j} a_{j} v_{j}\right)=a_{n} v_{1}+\sum_{j=1}^{n-1} a_{j} v_{j+1}+\sum_{j=n+1}^{\infty} a_{j} v_{j} .
$$

Then $U_{m}^{-1}\left(\sum_{j} a_{j} v_{j}\right)=\sum_{j=2}^{m} a_{j} v_{j-1}+a_{1} v_{m}+\sum_{j=m+1}^{\infty} a_{j} v_{j}$. Therefore, for $n<m$,

$$
\left(U_{m}^{-1} U_{n}\right)\left(\sum_{j} a_{j} v_{j}\right)=\sum_{j=1}^{n-1} a_{j} v_{j}+\sum_{j=n}^{m-1} a_{j+1} v_{j}+a_{n} v_{m}+\sum_{j=m+1}^{\infty} a_{j} v_{j},
$$

$$
\left(U_{m}^{-1} U_{n}\right)\left(\sum_{j} a_{j} v_{j}\right)-\left(\sum_{j} a_{j} v_{j}\right)=\sum_{j=n}^{m-1}\left(a_{j+1}-a_{j}\right) v_{j}+\left(a_{n}-a_{m}\right) v_{m} .
$$


However,

$$
\begin{aligned}
& \left\|\sum_{j=n}^{m-1}\left(a_{j+1}-a_{j}\right) v_{j}+\left(a_{n}-a_{m}\right) v_{m}\right\| \\
& \quad \leq\left\|\sum_{j=n}^{m-1} a_{j+1} v_{j}+a_{n} v_{m}\right\|+\left\|\sum_{j=n}^{m-1} a_{j} v_{j}+a_{m} v_{m}\right\| \\
& \quad \leq\left(\sum_{j=n}^{m-1}\left|\left(a_{j+1}\right)\right|^{2}+\left|a_{n}\right|^{2}\right)^{1 / 2}+\left(\sum_{j=n}^{m-1}\left|a_{j}\right|^{2}+\left|a_{m}\right|^{2}\right)^{1 / 2} \leq 2\left(\sum_{j=n}^{\infty}\left|a_{j}\right|^{2}\right)^{1 / 2} .
\end{aligned}
$$

This shows that $\left\{U_{n}\right\}_{n}$ is a Cauchy sequence, and

$$
U_{n}\left(\sum_{j} a_{j} v_{j}\right)-\sum_{j} a_{j} v_{j+1}=a_{n} v_{1}+\sum_{j=n+1}^{\infty}\left(a_{j}-a_{j-1}\right) v_{j}
$$

converges to zero as $n \rightarrow \infty$. So $\left\{U_{n}\right\}_{n}$ strongly (equivalently, weakly) converges to the operator

$$
P\left(\sum_{j} a_{j} v_{j}\right)=\sum_{j} a_{j} v_{j+1}
$$

which is valued in the subspace $\left\{v_{1}\right\}^{\perp}$ of $\mathcal{H}$. So $P$ is not unitary.

This concludes that $G$ is not complete.

\section{PROPOSITION 6.1}

The group of unitary operators $G \equiv U(\mathcal{H})$ is a T-type group and not strongly T-type; therefore it satisfies u-duality but not $i$-duality.

\section{EXAMPLE 2}

Let $G_{0} \equiv\left\{U_{g}\right\}$ be a group of unitary operators on some Hilbert space $\mathcal{H}$. Introduce the strong (resp., weak) topology on $G_{0}$; then $G_{0}$ becomes a topological group.

Take $b$-completion $G$ of $G_{0}$ in $J(\mathcal{H})$. Lemma 1.3(2) shows that $G \subset U(\mathcal{H})$. Therefore $G$ is a $b$-complete topological group.

A similar argument as for Lemma 6.1 leads to the fact that this $G$ has an SSUR.

Therefore $G$ is a T-type group and satisfies the weak Tannaka-type u-duality.

\section{EXAMPLE 3}

We consider a sequence of topological groups as

$$
G \supset G_{1} \supset G_{2} \supset \cdots \supset G_{n} \supset \cdots, \quad \bigcap G_{n}=\{e\} .
$$

Here for each $n, G_{n}$ is a closed normal subgroup of $G$ and the factor group $G^{(n)} \equiv G / G_{n}$ is a locally compact topological group. 
We give the topology on $G$ the projective limit topology of $\left\{G^{(n)}\right\}$, that is, the weakest topology for which the canonical map

$$
\varphi_{n}: G \longrightarrow G^{(n)}
$$

is continuous and open.

We call such a $G$ a projective limit group of $\left\{G^{(n)}\right\}$ and write

$$
G \equiv \underset{n}{\operatorname{Proj}-\lim }\left\{G^{(n)}\right\}
$$

Under such a situation, for any neighborhood $V$ of $e$ in $G$, there exists an $n$ and a neighborhood $V_{n}$ of $e$ in $G^{(n)}$ such that

$$
\left(\varphi_{n}\right)^{-1}\left(V_{n}\right) \subset V \text {. }
$$

Any locally compact group has an SSUR, and any unitary representation of $G^{(n)}$ gives naturally a unitary representation of $G$ through the canonical $\operatorname{map} \varphi_{n}$. So $G$ has an SSUR too.

Any element $g$ in $G$ corresponds to a sequence $\left\{g^{(n)}\left(\in G^{(n)}\right)\right\}_{n}$ such that

$$
g^{(n)}=\varphi_{n}(g),
$$

and, for any Cauchy filter base $\mathcal{F} \equiv\left\{F_{\alpha}\right\}$ on $G, \mathcal{F}_{n} \equiv\left\{\varphi_{n}\left(F_{\alpha}\right)\right\}$ gives also a Cauchy filter base on a locally compact group $G^{(n)}$ which is complete. So $\mathcal{F}$ converges in $G$ and $G$ is complete.

Thus we get the following.

PROPOSITION 6.2

The limit group $G \equiv \operatorname{Proj}_{-} \lim _{n}\left\{G^{(n)}\right\}$ is strongly-T-type; therefore it satisfies i-duality.

\section{EXAMPLE 4}

In the papers [7] and [8], we have shown that inductive limit groups $G \equiv$ $\lim _{n \rightarrow \infty} G_{n}$, where each $G_{n}$ is locally compact and $G_{n}$ is embedded as a closed subgroup into $G_{n+1}$ but not locally homeomorphic, satisfies the i-duality theorem. In this situation, $G$ is not locally compact.

So, this group gives an example which satisfies i-duality but not b-duality.

\section{EXAMPLE 5}

C. Chevalley gave a complexification of compact Lie groups in his book [1] using Tannaka duality for compact groups.

His argument is as follows. He gives a lemma for compact Lie groups.

\section{LEMMA 6.3}

A compact group is a Lie group if and only if it has a faithful finite-dimensional unitary representation $D \equiv\left\{\mathcal{H}, T_{g}\right\}$. 
By this lemma, $G$ is imbedded isomorphically into $\operatorname{GL}(d, \mathbf{C})$. We will write it as $G_{0}$. He considered the algebra $\mathfrak{F}$ of functions generated by all matrix elements $\mathfrak{F} \equiv\left\{\left\langle T_{g} u, v\right\rangle, \overline{\left\langle T_{g} u, v\right\rangle}(u, v \in \mathcal{H})\right\}$. That is, the same as $\mathfrak{F}$ is a ${ }^{*}$-algebra of functions generated by matrix elements of unitary representations through tensor products and direct sums of representations.

He considered the set $\left(G_{0}\right)^{\mathbf{C}}=\operatorname{Hom}(\mathfrak{F}, \mathbf{C})$ and showed that $\left(G_{0}\right)^{\mathbf{C}}$ must be an algebraic subgroup of $\mathrm{GL}(d, \mathbf{C})$ topologically isomorphic to $G \times \mathbf{R}^{\operatorname{dim} G}$.

We can extend any irreducible representation $\omega$ of $G$ to a representation $\widetilde{\omega} \equiv\left\{\mathcal{H}^{\omega}, T_{g_{0}}^{\omega}\right\}$ of $\left(G_{0}\right)^{\mathbf{C}}$. But it may not be a unitary representation of $\left(G_{0}\right)^{\mathbf{C}}$.

Any unitary representation $D$ of $G$ is decomposable to a discrete direct sum as $D \sim \sum_{\alpha}^{\oplus} \omega_{\alpha}$, and according to this decomposition, we can consider $\widetilde{D} \sim \sum_{\alpha}^{\oplus} \widetilde{\omega}_{\alpha}$.

On the space $\widetilde{\mathcal{H}}^{D} \equiv \sum_{\alpha}^{\oplus} \mathcal{H}^{\omega_{\alpha}}$, the operator $\left(\widetilde{T}_{g_{0}}^{D}\right)_{0}=\sum_{\alpha}^{\oplus} T_{g_{0}}^{\omega_{\alpha}}$ is closable and

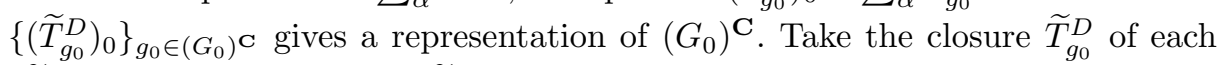
$\left(\widetilde{T}_{g_{0}}^{D}\right)_{0}$ on $\mathcal{H}^{D}$; then $\mathbf{T}^{D} \equiv\left\{\widetilde{T}_{g_{0}}^{D}\right\}_{g_{0} \in\left(G_{0}\right)^{\mathbf{C}}}$ is also a representation of $\left(G_{0}\right)^{\mathbf{C}}$.

Moreover, the relations of elements of the algebra $\mathfrak{F}$ shows that these representations of $\left(G_{0}\right)^{\mathbf{C}}$ satisfy (B-1)-(B-4) in Section 1.

Consequently, we see that $\mathbf{T}_{g_{0}} \equiv\left\{\widetilde{T}_{g_{0}}^{D}\right\}_{D \in \Omega}$ gives a cl-birepresentation.

This complexification of a compact Lie group is an example of a group for which b-duality is valid but c-duality is not valid.

\section{EXAMPLE 6}

A totally disconnected locally compact group gives an example for which cduality is valid, since it is a locally compact NOS-group.

Acknowledgments. The author would like to thank Professors Takeshi Hirai and Yoshiomi Nakagami who made many valuable comments.

\section{References}

[1] C. Chevalley, Theory of Lie Groups, I, Princeton Math. Ser. 8, Princeton Univ. Press, Princeton, 1946. MR 0015396.

[2] K. Saito, On a duality for locally compact groups, Tôhoku Math. J. (2) 20 (1968), 355-367. MR 0238997.

[3] N. Tatsuuma, A duality theorem for locally compact groups, J. Math. Kyoto Univ. 6 (1967), 187-293. MR 0217222.

[4] - "Duality theorem for locally compact groups and some related topics" in Algèbres d'opérateurs et leurs applications en physique mathématique (Marseille, 1977), Colloq. Internat. CNRS 274, CNRS, Paris, 1977, 387-408. MR 0560651.

[5] _ "Duality theorem for inductive limit group of direct product type" in Representation Theory and Analysis on Homogeneous Spaces, RIMS Kôkyûroku Bessatsu B7, Res. Inst. Math. Sci. (RIMS), Kyoto, 2008, 13-23. MR 2449443. 
[6]_ Duality theorem for inductive limit group (in Japanese), RIMS Kôkyûroku 1722 (2010), 48-67.

[7] N. Tatsuuma, A duality theorem for inductive limit group, preprint.

[8] N. Tatsuuma, H. Shimomura, and T. Hirai, On group topologies and unitary representations of inductive limits of topological groups and the case of the group of diffeomorphisms, J. Math. Kyoto Univ. 38 (1998), 551-578. MR 1661157.

[9] K. Yosida, Functional Analysis, reprint of the 6th (1980) ed., Classics Math., Springer, Berlin, 1995.

Nishinomiya City, Matsuoi-chô 10-8, 662-0076 Japan 